\title{
Effect of molecular conformations on the electronic transport in oxygen-substituted alkanethiol molecular junctions
}

Minglang Wang, Hao Wang, Guangping Zhang, Yongfeng Wang, Stefano Sanvito, and Shimin Hou

Citation: The Journal of Chemical Physics 148, 184703 (2018); doi: 10.1063/1.5025190

View online: https://doi.org/10.1063/1.5025190

View Table of Contents: http://aip.scitation.org/toc/jcp/148/18

Published by the American Institute of Physics

\section{Articles you may be interested in}

Perspective: Theory of quantum transport in molecular junctions

The Journal of Chemical Physics 148, 030901 (2018); 10.1063/1.5003306

Enhancing the conductivity of molecular electronic devices

The Journal of Chemical Physics 146, 092310 (2017); 10.1063/1.4972992

Temperature dependent tunneling conductance of single molecule junctions

The Journal of Chemical Physics 146, 092311 (2017); 10.1063/1.4973318

Perspective: Thermal and thermoelectric transport in molecular junctions

The Journal of Chemical Physics 146, 092201 (2017); 10.1063/1.4976982

The low-bias conducting mechanism of single-molecule junctions constructed with methylsulfide linker groups and gold electrodes

The Journal of Chemical Physics 147, 054702 (2017); 10.1063/1.4996745

Destructive quantum interference in electron transport: A reconciliation of the molecular orbital and the atomic orbital perspective

The Journal of Chemical Physics 146, 092308 (2017); 10.1063/1.4972572

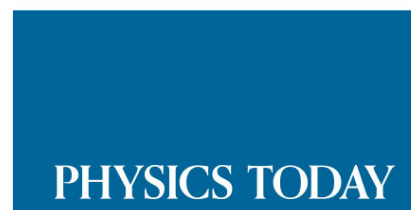

WHITEPAPERS
ADVANCED LIGHT CURE ADHESIVES

Take a closer look at what these environmentally friendly adhesive systems can do

\section{READ NOW}

PRESENTED BY

Q MASTERBOND。 


\title{
Effect of molecular conformations on the electronic transport in oxygen-substituted alkanethiol molecular junctions
}

\author{
Minglang Wang, ${ }^{1}$ Hao Wang, ${ }^{1}$ Guangping Zhang, ${ }^{2}$ Yongfeng Wang, ${ }^{1,3}$ Stefano Sanvito, ${ }^{4}$ \\ and Shimin $\mathrm{Hou}^{1,3, \mathrm{a})}$ \\ ${ }^{1}$ Key Laboratory for the Physics and Chemistry of Nanodevices, Department of Electronics, \\ Peking University, Beijing 100871, China \\ ${ }^{2}$ School of Physics and Electronics, Shandong Normal University, Jinan 250358, China \\ ${ }^{3}$ Beida Information Research (BIR), Tianjin 300457, China \\ ${ }^{4}$ School of Physics, AMBER and CRANN Institute, Trinity College, Dublin 2, Ireland
}

(Received 7 February 2018; accepted 13 April 2018; published online 14 May 2018)

\begin{abstract}
The relationship between the molecular structure and the electronic transport properties of molecular junctions based on thiol-terminated oligoethers, which are obtained by replacing every third methylene unit in the corresponding alkanethiols with an oxygen atom, is investigated by employing the non-equilibrium Green's function formalism combined with density functional theory. Our calculations show that the low-bias conductance depends strongly on the conformation of the oligoethers in the junction. Specifically, in the cases of trans-extended conformation, the oxygen-dominated transmission peaks are very sharp and well below the Fermi energy, $E_{\mathrm{F}}$, thus hardly affect the transmission around $E_{\mathrm{F}}$; the $\mathrm{Au}-\mathrm{S}$ interface hybrid states couple with $\sigma$-bonds in the molecular backbone forming the conduction channel at $E_{\mathrm{F}}$, resulting in a conductance decay against the molecular length close to that for alkanethiols. By contrast, for junctions with oligoethers in helical conformations, some $\pi$-type oxygen orbitals coupling with the $\mathrm{Au}-\mathrm{S}$ interface hybrid states contribute to the transmission around $E_{\mathrm{F}}$. The molecule-electrode electronic coupling is also enhanced at the non-thiol side due to the specific spatial orientation introduced by the twist of the molecular backbone. This leads to a much smaller conductance decay constant. Our findings highlight the important role of the molecular conformation of oligoethers in their electronic transport properties and are also helpful for the design of molecular wires with heteroatom-substituted alkanethiols. Published by AIP Publishing. https://doi.org/10.1063/1.5025190
\end{abstract}

\section{INTRODUCTION}

Establishing relationships between the electronic transport properties of single-molecule junctions and their atomic structure is an important and challenging task in the field of molecular electronics. ${ }^{1-8}$ Besides the metal electrodes and the anchor groups, the molecular structure and the ensuing electronic structure of the central molecules are vital factors for determining the current-voltage characteristics of molecular junctions. So far, a large amount of molecules with different kinds of backbones have been systematically studied. These include saturated hydrocarbons, ${ }^{9-12}$ aromatic molecules, ${ }^{13-15}$ porphyrins, ${ }^{16-18}$ and so on. Among these, alkanethiol-based junctions have attracted significant attention because of their simple molecular structure and the high reproducibility of the experimental measurements. ${ }^{10-12,19-25}$ By exploring the low-bias junction conductance as a function of the molecular length, it is generally accepted that off-resonance tunneling is the conducting mechanism of alkanethiol-based junctions. In order to further tune the electronic transport properties of alkanethiol-based molecular junctions, heteroatoms are introduced to probe the effects of local atomic and electronic

a)Author to whom correspondence should be addressed: smhou@pku.edu.cn changes on the junction conductance. For example, an alkanethiol molecule is changed into a thiol-terminated oligoether molecule with similar length when every third methylene $\left(-\mathrm{CH}_{2}-\right)$ unit is substituted by an oxygen atom. Scullion et al. measured the low-bias conductance of ether and alkane chains connected to gold electrodes with thiol groups using the scanning tunneling microscopy $\mathrm{I}(\mathrm{s})$ method and found that the conductance values of the $\mathrm{Au}-\mathrm{S}\left(\mathrm{CH}_{2}\right)_{4} \mathrm{O}\left(\mathrm{CH}_{2}\right)_{4}$ $\mathrm{S}-\mathrm{Au}$ and $\mathrm{Au}-\mathrm{S}\left(\mathrm{CH}_{2} \mathrm{CH}_{2} \mathrm{O}\right)_{2}\left(\mathrm{CH}_{2}\right)_{2} \mathrm{~S}-\mathrm{Au}$ junctions are higher than those of the length-matched $\mathrm{Au}-\mathrm{S}\left(\mathrm{CH}_{2}\right)_{9} \mathrm{~S}-\mathrm{Au}$ and $\mathrm{Au}-\mathrm{S}\left(\mathrm{CH}_{2}\right)_{8} \mathrm{~S}-\mathrm{Au}$ junctions. ${ }^{26}$ By contrast, Wierzbinski et al. and Xie et al. both reported that the measured conductance values of the oligoethers are lower than those of the alkane chains with the same length ${ }^{27,28}$ and that the conductance decay constant $\beta$ of the oligoethers is slightly higher than that of the alkane chains. ${ }^{28}$ Interestingly, a more recent experimental observation from Baghbanzadeh et al. revealed an anomalously small conductance decay in oligoethers with $\beta=0.29 \pm 0.02 /$ atom, a much lower value than that of alkanes $\beta=0.94 \pm 0.02 /$ atom. ${ }^{29-31}$ Concerning the interpretation of the conduction mechanism, Scullion et al. and Baghbanzadeh et al. ascribed the high conductance values and their small decay in oligoethers to the high-energy occupied orbitals associated with the lone-pair electrons on oxygen that are believed to decrease the tunneling barrier height. ${ }^{26,29}$ By 
contrast, Wierzbinski et al. and Xie et al. proposed that the lower conductance values of oligoethers are traced to the more localized oxygen-related molecular orbitals. ${ }^{27,28}$ Therefore, there is still a debate on how the oxygen substitution in saturated alkane chains affects the molecular conductance.

It is well-known that the conductance measured for molecules can be affected significantly by the moleculeelectrode binding motifs and by the conformation of the central molecule in the junction. However, resolving the molecular structure of a junction from experiments is still very difficult, a fact that largely hinders further exploration of the structure-property relationship in molecular junctions. ${ }^{32}$ Computational simulations have the advantage of complementing this deficiency since they can easily explore the effects of the contact geometries and molecular conformations. Here, we theoretically study the electronic transport properties of thiol-terminated oligoether junctions employing the non-equilibrium Green's function formalism combined with density functional theory (the NEGF+DFT approach), ${ }^{33-41}$ especially focusing on the influence of the molecular conformation of oligoethers on the conductance decay against the molecular length. Our calculations show that the conductance of molecular junctions with oligoethers in the helical conformation has a much smaller decay constant than that of oligoethers in the trans-extended conformation. Further analysis of the electronic transmission spectra as well as the eigenchannel reveals that the $\mathrm{Au}-\mathrm{S}$ interface hybrid states dominate the electron tunneling around the Fermi energy, $E_{\mathrm{F}}$. However, the tunneling efficiency strongly depends on the conformation of the oligoethers. More specifically, for molecular junctions with oligoethers in the trans-extended conformation, the transmission peaks dominated by the $\pi$-type oxygen lone-pair states have sharp structures and are positioned well below $E_{\mathrm{F}}$. The conduction channel at $E_{\mathrm{F}}$ is then formed by the $\mathrm{Au}-\mathrm{S}$ interface hybrid states coupling to the $\sigma$-bonds along the molecular backbone. Thus, their conductance decay constant is almost the same as that for alkanethiols. By contrast, when oligoethers adopt helical conformations, some $\pi$-type oxygen orbitals couple with the $\mathrm{Au}-\mathrm{S}$ interfacial hybrid states contributing to the transmission around $E_{\mathrm{F}}$, due to the specific spatial orientation among the oxygen and sulfur atoms and the shortened $\mathrm{S}-\mathrm{O}$ and $\mathrm{O}-\mathrm{O}$ distances. The electronic coupling between longer oligoether molecules and the gold electrode at the non-thiol side is also promoted by the direct $\mathrm{Au}-\mathrm{O}$ interaction. As a result, a much smaller conductance decay constant occurs for the oligoethers in helical conformations.

\section{THEORETICAL METHOD AND COMPUTATIONAL DETAILS}

We employ the SIESTA software package to investigate the atomic and electronic structures of Au-molecule-Au molecular junctions and the SMEAGOL (Spin and Molecular Electronics Algorithm on a Generalized atomic Orbital Landscape) code to study their electronic transport properties. ${ }^{40-42}$ SIESTA is an efficient DFT package for numerical simulations of a large system with affordable resources, in which the wave functions of valence electrons are expanded over a finiterange numerical basis set and the core electrons are described by norm-conserving Troullier-Martins pseudopotentials. ${ }^{42,43}$ While a double-zeta plus polarization (DZP) basis set is used for $\mathrm{H}, \mathrm{C}, \mathrm{O}$, and $\mathrm{S}$ atoms, two different types of basis functions are used for $\mathrm{Au}$, respectively, in the bulk and at the surface. In more detail, a DZP basis set augmented with diffuse functions is used for the Au surface atoms, while a singlezeta plus polarization (SZP) basis is used for the bulk. The exchange-correlation functional is treated at the level of the generalized gradient approximation (GGA) within the PerdewBurke-Ernzerhof (PBE) formulation. ${ }^{44}$ An equivalent cutoff of 200.0 Ry is taken for the real space grid integration. Geometry optimization is performed by standard conjugate gradient until all the atomic forces are smaller than $0.03 \mathrm{eV} \AA^{-1}$.

SMEAGOL is a practical implementation of the NEGF+DFT approach, which uses SIESTA as the DFT platform. ${ }^{40,41}$ Periodic boundary conditions are applied in the plane transverse to the transport direction, while the unit cell of the extended molecule includes the central molecule and ten atomic layers of the gold electrodes with a $(3 \times 3)$ supercell. The charge density is integrated over 24 energy points along the semi-circle, 24 points along the line in the complex plane, while 24 poles are used for the Fermi function (the electronic temperature is $25 \mathrm{meV}$ ). The transmission coefficient $T(E)$ of the molecular junction is evaluated as

$$
T(E)=\frac{1}{\Omega_{2 D B Z}} \int_{2 D B Z} T(\vec{k} ; E) d \vec{k},
$$

where $\Omega_{2 \mathrm{DBZ}}$ is the area of the two-dimensional Brillouin zone (2DBZ) in the transverse directions (orthogonal to the transport direction). The $k$-dependent transmission coefficient $T(\vec{k} ; E)$ is obtained as

$$
T(\vec{k} ; E)=\operatorname{Tr}\left[\Gamma_{L} G_{M}^{R} \Gamma_{R} G_{M}^{R+}\right],
$$

where $G_{M}^{R}$ is the retarded Green's function matrix of the extended molecule and $\Gamma_{\mathrm{L}}\left(\Gamma_{\mathrm{R}}\right)$ is the broadening function matrix describing the interaction of the extended molecule with the left-hand (right-hand) side electrode. Here, we calculate the transmission coefficient by sampling $4 \times 4 \mathrm{k}$-points in the transverse 2DBZ.

\section{RESULTS AND DISCUSSION}

We start our studies with the investigation of the atomic and electronic structures of isolated oligoether molecules in the trans-extended conformation. With a thiol group terminated at one end, these oligoethers are denoted as trans- $\mathrm{HS}\left(\mathrm{CH}_{2} \mathrm{CH}_{2} \mathrm{O}\right)_{\mathrm{n}} \mathrm{CH}_{3}$, where $\mathrm{n}$ is the number of the $\mathrm{CH}_{2} \mathrm{CH}_{2} \mathrm{O}$ units in the molecular backbone. Taking the trans$\mathrm{HS}\left(\mathrm{CH}_{2} \mathrm{CH}_{2} \mathrm{O}\right)_{3} \mathrm{CH}_{3}$ molecule as a representative of this family, its optimized atomic structure is shown in Fig. 1(a). As we can see, all of the non-hydrogen atoms including S, C, and $\mathrm{O}$ are coplanar. The $\mathrm{C}-\mathrm{O}$ bond lengths in the backbone are optimized to be $1.43 \AA$, close to the $\mathrm{C}-\mathrm{C}$ bond lengths $(1.53 \AA)$ in the corresponding trans- $\mathrm{HS}\left(\mathrm{CH}_{2}\right)_{9} \mathrm{CH}_{3}$ molecule. At the same time, the values of the $\angle \mathrm{C}-\mathrm{O}-\mathrm{C}$ bond angles in trans- $\mathrm{HS}\left(\mathrm{CH}_{2} \mathrm{CH}_{2} \mathrm{O}\right)_{3} \mathrm{CH}_{3}$ are nearly the same as those 
(a)
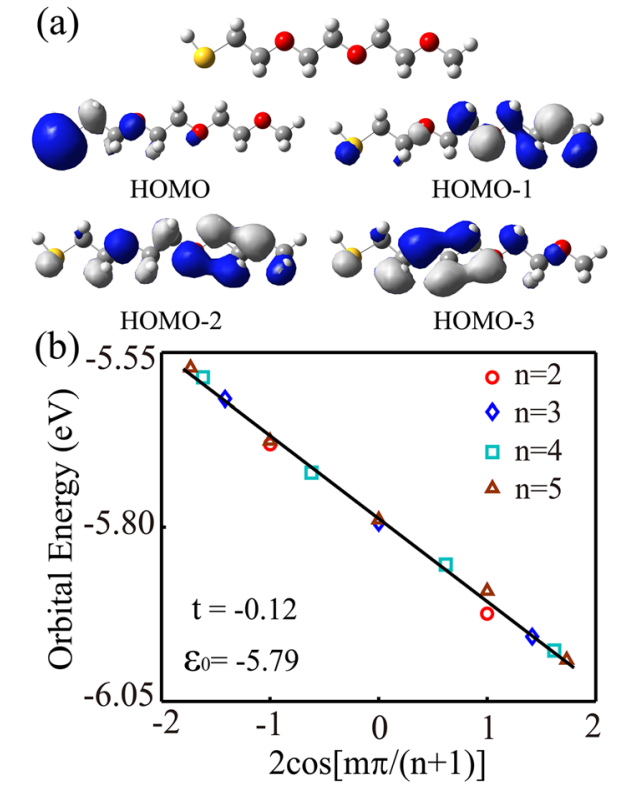

FIG. 1. (a) The optimized atomic structure and the occupied frontier molecular orbitals of an isolated trans- $\mathrm{HS}\left(\mathrm{CH}_{2} \mathrm{CH}_{2} \mathrm{O}\right)_{3} \mathrm{CH}_{3}$ molecule. (b) Linear fit for the orbital energies of the oxygen lone pairs of isolated trans$\mathrm{HS}\left(\mathrm{CH}_{2} \mathrm{CH}_{2} \mathrm{O}\right)_{n} \mathrm{CH}_{3}$ molecules with $\mathrm{n}=2,3,4$, and 5. In (a), light gray, gray, red, and yellow spheres are, respectively, hydrogen, carbon, oxygen, and sulfur atoms.

of the $\angle \mathrm{C}-\mathrm{C}-\mathrm{C}$ bond angles in trans- $\mathrm{HS}\left(\mathrm{CH}_{2}\right)_{9} \mathrm{CH}_{3}$, both of which are around $112^{\circ}$. Similar results are obtained for the $\mathrm{C}-\mathrm{O}$ bond lengths and the $\angle \mathrm{C}-\mathrm{O}-\mathrm{C}$ bond angles in other trans- $\mathrm{HS}\left(\mathrm{CH}_{2} \mathrm{CH}_{2} \mathrm{O}\right)_{\mathrm{n}} \mathrm{CH}_{3}$ molecules with $\mathrm{n}=2$, 4, and 5 . Therefore, the molecular lengths of oligoethers are almost equal to those of the corresponding alkanethiols.

The highest occupied molecular orbital (HOMO) of trans$\mathrm{HS}\left(\mathrm{CH}_{2} \mathrm{CH}_{2} \mathrm{O}\right)_{3} \mathrm{CH}_{3}$ is dominated by the $3 \mathrm{p}$ atomic orbital of the terminal sulfur atom [see Fig. 1(a)], ${ }^{26-28}$ which is perpendicular to the plane of the molecular backbone. The $\pi$-type electronic coupling between the S $3 p$ atomic orbital and the adjacent $\mathrm{O} 2 \mathrm{p}$ atomic orbital is rather weak due to their energy mismatch and the isolation produced by the two intermediate methylene groups. By contrast, the HOMO-1, HOMO-2, and HOMO-3 orbitals are delocalized along the three oxygen atoms, ${ }^{26,27}$ which are mainly contributed by the oxygen lone pairs and thus have an obvious $\pi$-conjugation feature. Occupied frontier molecular orbitals with the same bonding characteristics are also observed in other trans- $\mathrm{HS}\left(\mathrm{CH}_{2} \mathrm{CH}_{2} \mathrm{O}\right)_{n} \mathrm{CH}_{3}$ molecules with $\mathrm{n}=2,4$, and 5: the HOMO is predominately distributed on the sulfur atom of the thiol group, while the molecular orbitals going from HOMO-1 to HOMO-n are $\pi$-type and dominated by the oxygen lone pairs of electrons. These oxygen-dominated molecular levels are separated with almost equal energy intervals and can be well described by a one-dimensional $\mathrm{n}$-site infinite potential well $\varepsilon_{\mathrm{m}}=\varepsilon_{0}$ $+2 t \cos (m \pi /(n+1)),{ }^{45}$ where the index $m=1,2, \ldots, n$ labels the molecular levels and the on-site energy and the hopping integral are, respectively, fitted to be $\varepsilon_{0}=-5.79 \mathrm{eV}$ and $\mathrm{t}=-0.12 \mathrm{eV}$ [see Fig. 1(b)]. This illustrates that each oxygen atom in the backbone makes an equal contribution to these $\pi$-type molecular orbitals and further confirms the weak $\pi$-type electronic coupling between the thiol group and the substituted oxygen atoms.

Then we investigate the electronic transport properties of oligoethers in the trans-extended conformation. The optimized atomic structure of the $\mathrm{Au}$-trans- $\mathrm{S}\left(\mathrm{CH}_{2} \mathrm{CH}_{2} \mathrm{O}\right)_{3} \mathrm{CH}_{3}-$ Au molecular junction is shown in Fig. 2(a). Here the molecule is assumed to chemically bind at the hollow site of the $\mathrm{Au}(111)$ surface through $\mathrm{Au}-\mathrm{S}$ covalent bonds at the left side and is physically connected to the right gold electrode through the terminal methyl group. The molecular backbone is placed in the $\mathrm{yOz}$ plane; the $\mathrm{Au}-\mathrm{S}$ bond lengths and the distance between the rightmost hydrogen atom in the terminal methyl group and the right electrode surface are, respectively, optimized to be $2.51 \AA$ and $2.64 \AA$. The equilibrium transmission spectrum plotted in a semi-logarithmic scale is presented in Fig. 2(b). The first transmission peak below the Fermi energy appears at
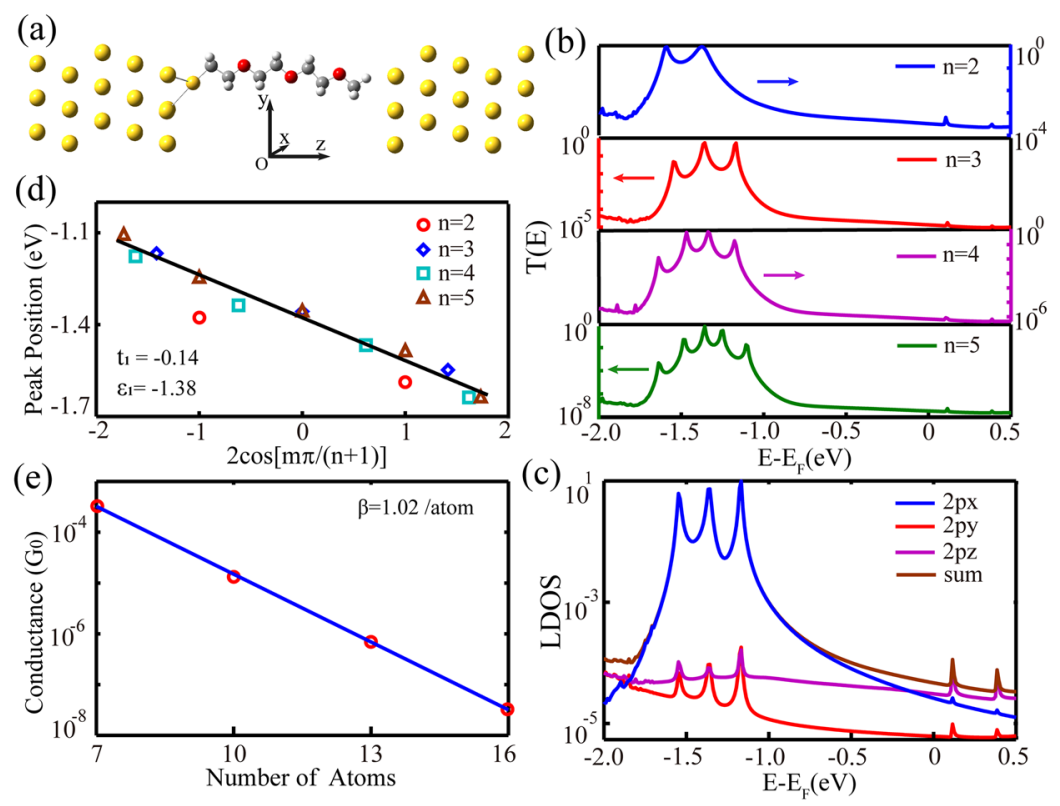

FIG. 2. (a) The optimized atomic structure of the $\mathrm{Au}$-trans-S( $\left.\mathrm{CH}_{2} \mathrm{CH}_{2} \mathrm{O}\right)_{3} \mathrm{CH}_{3}-\mathrm{Au}$ molecular junction, (b) the equilibrium transmission spectra of the $\mathrm{Au}-$ trans- $\mathrm{S}\left(\mathrm{CH}_{2} \mathrm{CH}_{2} \mathrm{O}\right)_{\mathrm{n}} \mathrm{CH}_{3}-\mathrm{Au}$ molecular junctions with $\mathrm{n}=2,3,4$, and 5, (c) the LDOS of the Au-trans$\mathrm{S}\left(\mathrm{CH}_{2} \mathrm{CH}_{2} \mathrm{O}\right)_{3} \mathrm{CH}_{3}-\mathrm{Au}$ molecular junction projected onto the oxygen $2 \mathrm{p}$ atomic orbitals, (d) linear fit for the oxygen-dominated peak positions in the $\mathrm{Au}-$ trans$\mathrm{S}\left(\mathrm{CH}_{2} \mathrm{CH}_{2} \mathrm{O}\right)_{\mathrm{n}} \mathrm{CH}_{3}-\mathrm{Au}$ molecular junctions with $\mathrm{n}=3$, 4 , and 5, and (e) linear fit for the conductance decay constant of the $\mathrm{Au}$-trans- $\mathrm{S}\left(\mathrm{CH}_{2} \mathrm{CH}_{2} \mathrm{O}\right)_{\mathrm{n}} \mathrm{CH}_{3}-\mathrm{Au}$ molecular junctions with $\mathrm{n}=2,3,4$, and 5 . 
$-1.17 \mathrm{eV}$ and decays rapidly toward $E_{\mathrm{F}}$; other two transmission peaks are centered at $-1.36 \mathrm{eV}$ and $-1.55 \mathrm{eV}$, respectively. By inspecting the local density of states (LDOSs) projected onto the oxygen atoms [see Fig. 2(c)], one can see that the O 2px atomic orbital contributes three large LDOS peaks centered at $-1.17 \mathrm{eV},-1.35 \mathrm{eV}$, and $-1.56 \mathrm{eV}$, in excellent agreement with the positions of the three transmission peaks. This indicates that these three transmission peaks are dominated by oxygen lone pairs of electrons.

Very similar junction structures are obtained for other trans- $\mathrm{HS}\left(\mathrm{CH}_{2} \mathrm{CH}_{2} \mathrm{O}\right)_{\mathrm{n}} \mathrm{CH}_{3}$ molecules with $\mathrm{n}=2,4$, and 5, and the overall shape of their equilibrium transmission spectra is almost identical to that of the Au-trans- $\mathrm{S}\left(\mathrm{CH}_{2} \mathrm{CH}_{2} \mathrm{O}\right)_{3} \mathrm{CH}_{3}-$ $\mathrm{Au}$ junction except that the number of the transmission peaks below $E_{\mathrm{F}}$ equals to that of the oxygen atoms in the molecular backbone [see Fig. 2(b)]. In Fig. 2(d), we fit the peak positions (ع) of the Au-trans- $\mathrm{S}\left(\mathrm{CH}_{2} \mathrm{CH}_{2} \mathrm{O}\right)_{\mathrm{n}} \mathrm{CH}_{3}-\mathrm{Au}$ junctions with $\mathrm{n}=3,4$, and 5 as a function of the number of oxygen atoms and find that the relation $\varepsilon=\varepsilon_{1}+2 \mathrm{t}_{1} \cos (\mathrm{m} \pi /(\mathrm{n}+1))$ still holds in the junctions with $\varepsilon_{1}=-1.38 \mathrm{eV}$ and $\mathrm{t}_{1}=-0.14 \mathrm{eV}$, indicating that these $\pi$-type molecular orbitals dominated by the oxygen lone pairs of electrons only couple weakly to the electronic states of gold electrodes. Extrapolating the number of oxygen atoms in the molecular backbone to infinity (an infinite chain), the first transmission peak (the band edge) will be up shifted to $-1.10 \mathrm{eV}$, but it is still much lower than the Fermi energy.

The low-bias junction conductance that is defined as the product of the quantum conductance, $\mathrm{G}_{0}=2 \mathrm{e}^{2} / \mathrm{h}$ ( $\mathrm{e}$ is the electron charge and $\mathrm{h}$ is the Planck constant), and the transmission coefficient at $E_{\mathrm{F}}$ demonstrates an exponential decay against the molecular length [see Fig. 2(e)], following the form $\mathrm{G}=\mathrm{G}_{\mathrm{c}} \exp (-\beta N)$, in which $\mathrm{G}_{\mathrm{c}}$ is the effective contact conductance and $\mathrm{N}$ is the number of non-hydrogen atoms in the molecular backbone. The conductance decay constant $\beta$ is fitted to be $1.02 /$ atom, very close to the accepted value of $\beta=0.94 /$ atom for alkanethiols. ${ }^{30,31}$

In order to get more insight into the nature of the transmission around $E_{\mathrm{F}}$ and validate the contribution of the oxygen atoms to the transmission peaks below $E_{\mathrm{F}}$, the eigenchannels of the Au-trans- $\mathrm{S}\left(\mathrm{CH}_{2} \mathrm{CH}_{2} \mathrm{O}\right)_{3} \mathrm{CH}_{3}-\mathrm{Au}$ junction are calculated at $E_{\mathrm{F}},-1.17 \mathrm{eV},-1.36 \mathrm{eV}$, and $-1.55 \mathrm{eV}$ (see Fig. 3). ${ }^{46,47}$ One can notice that the eigenchannels at the energies $-1.17 \mathrm{eV}$, $-1.36 \mathrm{eV}$, and $-1.55 \mathrm{eV}$ shown in Figs. 3(b)-3(d) are delocalized over the molecular backbone. By comparing these three eigenchannels with the HOMO-1, HOMO-2, and HOMO-3 orbitals of the isolated trans- $\mathrm{HS}\left(\mathrm{CH}_{2} \mathrm{CH}_{2} \mathrm{O}\right)_{3} \mathrm{CH}_{3}$ molecule [see Fig. 1(a)], we can find that these three transmission peaks are indeed dominated by the $\pi$-type oxygen lone-pair orbitals. By contrast, the eigenchannel at $E_{\mathrm{F}}$ has a dominant weight on the left terminal sulfur atom as shown in Fig. 3(a), indicating that it is mainly governed by the interface hybrid states formed between the anchoring sulfur atom and the surface gold atoms. When the isovalue at which the eigenchannel isosurface is drawn is decreased from $2 \times 10^{-4}$ bohrs $^{-3}$ to $2 \times 10^{-5}$ bohrs $^{-3}$, this eigenchannel shows a decay along the molecular backbone through $\mathrm{S}-\mathrm{C}, \mathrm{C}-\mathrm{C}$, and $\mathrm{C}-\mathrm{O} \sigma$-bonds. Therefore, the $\pi$-type oxygen lone-pair orbitals only make negligible contributions to the transmission around $E_{\mathrm{F}}$ due to their weak coupling to

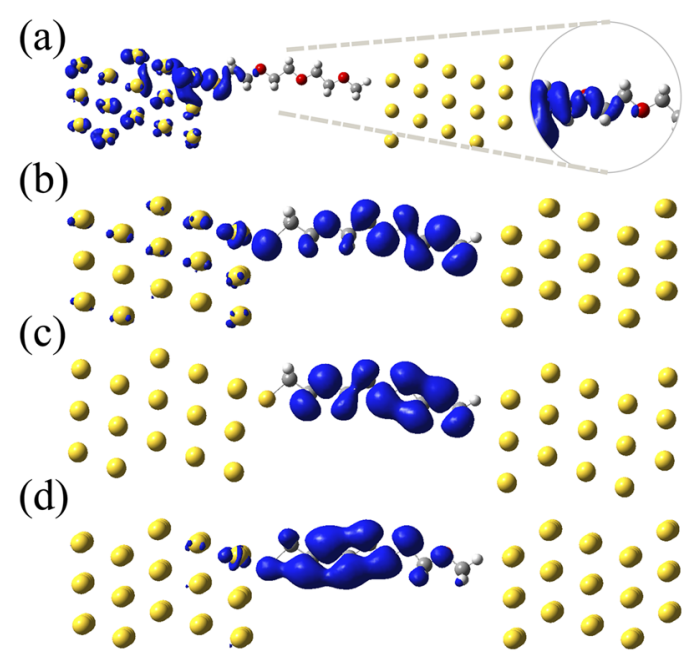

FIG. 3. Eigenchannels of the Au-trans-S( $\left.\mathrm{CH}_{2} \mathrm{CH}_{2} \mathrm{O}\right)_{3} \mathrm{CH}_{3}-\mathrm{Au}$ molecular junction calculated at $\mathrm{E}_{\mathrm{F}}(\mathrm{a}),-1.17 \mathrm{eV}(\mathrm{b}),-1.36 \mathrm{eV}$ (c), and $-1.55 \mathrm{eV}$ (d). The isovalue is set to be $2 \times 10^{-4}$ bohrs $^{-3}$, but is decreased to $2 \times 10^{-5}$ bohrs $^{-3}$ in the inset of (a).

the gold electrodes (sharp peak structures) and the low-lying energy positions (more than $1.10 \mathrm{eV}$ below $E_{\mathrm{F}}$ ). This also explains why the calculated conductance decay constant for oligoethers in the trans-extended conformation is very close to that of alkanes.

It is worth noting that although our calculated conductance decay constant $\beta$ for $\mathrm{Au}$-trans- $\mathrm{S}\left(\mathrm{CH}_{2} \mathrm{CH}_{2} \mathrm{O}\right)_{n} \mathrm{CH}_{3}-$ $\mathrm{Au}$ is much larger than that experimentally measured by Baghbanzadeh et al. ${ }^{29}$ it is well consistent with that reported by Xie $e t a l .{ }^{28}$ where oligoethers are bridged to the gold electrodes through thiol groups at both sides. In order to test the effects of different anchoring groups between our junction models and the experiments, the electronic transport properties of oligoethers connected to the gold electrode through thiol groups at both sides are also investigated. These junctions are termed $\mathrm{Au}$-trans- $\mathrm{S}\left(\mathrm{CH}_{2} \mathrm{CH}_{2} \mathrm{O}\right)_{\mathrm{n}} \mathrm{CH}_{2} \mathrm{CH}_{2} \mathrm{~S}-\mathrm{Au}$ with $\mathrm{n}=2$, 3 , and 4. The optimized atomic structure of the Au-trans$\mathrm{S}\left(\mathrm{CH}_{2} \mathrm{CH}_{2} \mathrm{O}\right)_{3} \mathrm{CH}_{2} \mathrm{CH}_{2} \mathrm{~S}-\mathrm{Au}$ molecule junction is shown in Fig. 4(a), and the equilibrium transmission spectra of the junctions with $n=2,3$, and 4 are presented in Fig. 4(b). Clearly, the replacement of the terminal methyl group with thiol does not change the overall shape of the transmission spectra, but indeed enhances the coupling strength of oligoethers with the right gold electrode. This results in a largely increased junction transmission and in a slight shift of the peak positions dominated by the oxygen lone-pair orbitals. Moreover, we find that the transmission around $E_{\mathrm{F}}$ is still dominated by the electron tunneling of the Au-S interface hybrid states through $\sigma$-bonds of the molecular backbone, which in turn manifests a conductance decay constant $\beta=1.01 /$ atom [see Fig. 4(c)]. Therefore, the low-bias conduction mechanism of the Au-trans- $\mathrm{S}\left(\mathrm{CH}_{2} \mathrm{CH}_{2} \mathrm{O}\right)_{n} \mathrm{CH}_{3}-\mathrm{Au}$ junctions is the same as that of the Au-trans-S $\left(\mathrm{CH}_{2} \mathrm{CH}_{2} \mathrm{O}\right)_{n} \mathrm{CH}_{2} \mathrm{CH}_{2} \mathrm{~S}-$ $\mathrm{Au}$ junctions, regardless of the detailed contact configuration between the oligoether molecule and the right gold electrode.

To date, the underlying mechanism for the anomalously small conductance decay constant in oligoethers observed 
(a)
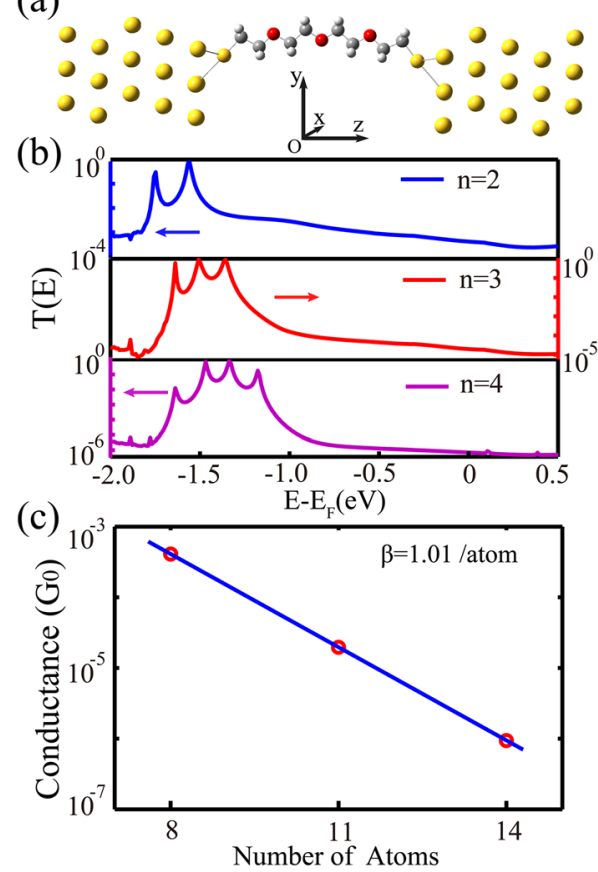

FIG. 4. The optimized atomic structure (a) of the Au-trans$\mathrm{S}\left(\mathrm{CH}_{2} \mathrm{CH}_{2} \mathrm{O}\right)_{3} \mathrm{CH}_{2} \mathrm{CH}_{2} \mathrm{~S}-\mathrm{Au}$ molecular junction, the equilibrium transmission spectra (b) and the linear fit for the conductance decay constant (c) of the Au-trans- $\mathrm{S}\left(\mathrm{CH}_{2} \mathrm{CH}_{2} \mathrm{O}\right)_{n} \mathrm{CH}_{2} \mathrm{CH}_{2} \mathrm{~S}-\mathrm{Au}$ molecular junctions with $\mathrm{n}=2,3$, and 4 .

experimentally by Baghbanzadeh et al. still remains unexplained. ${ }^{29}$ It is noticed that the measured thickness values of the self-assembled oligoether monolayers are much smaller than the molecular lengths calculated for trans-extended oligoethers. Therefore, oligoethers with helical conformations may exist in the junctions. In what follows, we investigate the atomic and electronic structures and the electron transport properties of oligoethers with helical conformations. These are denoted as helical- $\mathrm{HS}\left(\mathrm{CH}_{2} \mathrm{CH}_{2} \mathrm{O}\right)_{n} \mathrm{CH}_{3}$. Figure 5(a) shows the optimized atomic structure and the occupied frontier molecular orbitals of the isolated helical- $\mathrm{HS}\left(\mathrm{CH}_{2} \mathrm{CH}_{2} \mathrm{O}\right)_{3} \mathrm{CH}_{3}$ molecule. When compared with trans- $\mathrm{HS}\left(\mathrm{CH}_{2} \mathrm{CH}_{2} \mathrm{O}\right)_{3} \mathrm{CH}_{3}$, the changes of the $\mathrm{C}-\mathrm{O}$ bond lengths and the $\angle \mathrm{C}-\mathrm{O}-\mathrm{C}$ bond angles are, respectively, less than $0.01 \AA$ and $2^{\circ}$. However, the $\mathrm{S}-\mathrm{O}$ and $\mathrm{O}-\mathrm{O}$ distances are shortened by $\sim 1 \AA$, and the spatial orientation among the oxygen and sulfur atoms is also changed significantly, leading to great modifications of the occupied frontier molecular orbitals. Qualitatively, these oxygen lone-pair states are no longer pure $\pi$-type molecular orbitals because the reduced symmetry of the helical conformation causes some $\pi-\sigma$ hybridization. Quantitatively, these oxygen lone-pair states not only shift to higher energies but also distribute over a wider energy range [see Fig. 5(b)], illustrating that the electronic coupling between the oxygen atoms is enhanced. The HOMO is also more delocalized due to the enhanced interaction between the sulfur atom and the adjacent oxygen atom. Similar results are also obtained for other helical-HS $\left(\mathrm{CH}_{2} \mathrm{CH}_{2} \mathrm{O}\right)_{n} \mathrm{CH}_{3}$ molecules with $\mathrm{n}=4$ and 5 .

The optimized atomic structures of $\mathrm{Au}$-helical$\mathrm{S}\left(\mathrm{CH}_{2} \mathrm{CH}_{2} \mathrm{O}\right)_{\mathrm{n}} \mathrm{CH}_{3}-\mathrm{Au}$ molecular junctions with $\mathrm{n}=3$, 4, and (a)
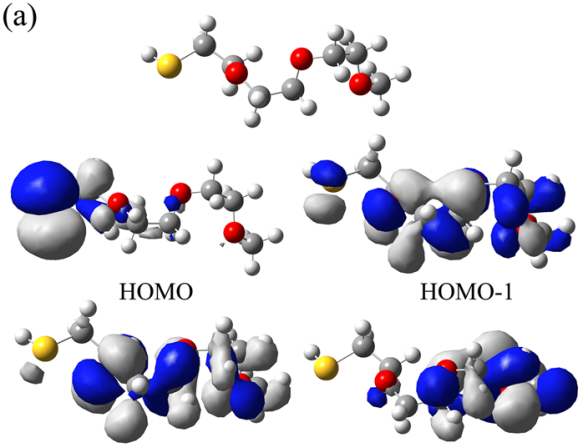

(b)

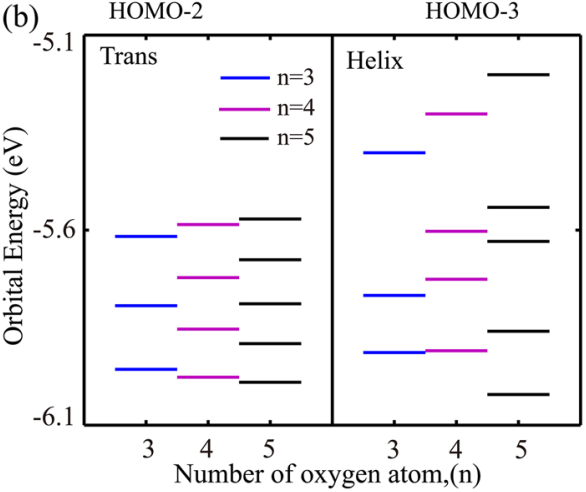

FIG. 5. (a) The optimized atomic structure and the occupied frontier molecular orbitals of an isolated helical- $\mathrm{HS}\left(\mathrm{CH}_{2} \mathrm{CH}_{2} \mathrm{O}\right)_{3} \mathrm{CH}_{3}$ molecule and (b) comparison for the orbital energies of oxygen lone pairs of isolated trans-and helical- $\mathrm{HS}\left(\mathrm{CH}_{2} \mathrm{CH}_{2} \mathrm{O}\right)_{\mathrm{n}} \mathrm{CH}_{3}$ molecules with $\mathrm{n}=3,4$, and 5 .

5 are shown in Fig. 6(a). At the left molecule-electrode interface of these three molecular junctions, the $\mathrm{Au}-\mathrm{S}$ bond lengths are optimized to be $2.53 \AA$ very close to those in the corresponding $\mathrm{Au}-$ trans- $\mathrm{S}\left(\mathrm{CH}_{2} \mathrm{CH}_{2} \mathrm{O}\right)_{\mathrm{n}} \mathrm{CH}_{3}-\mathrm{Au}$ junctions. By contrast, the right molecule-electrode interfaces are markedly different: both the terminal methyl group and its neighboring $\mathrm{CH}_{2} \mathrm{CH}_{2} \mathrm{O}$ group face the right gold electrode in the $\mathrm{Au}$ helical-S( $\left(\mathrm{CH}_{2} \mathrm{CH}_{2} \mathrm{O}\right)_{\mathrm{n}} \mathrm{CH}_{3}$-Au molecular junctions with $\mathrm{n}=4$ and 5 while it is still the terminal methyl group that points to the right electrode surface in the Au-helical- $\mathrm{S}\left(\mathrm{CH}_{2} \mathrm{CH}_{2} \mathrm{O}\right)_{3} \mathrm{CH}_{3}-$ $\mathrm{Au}$ junction. Noticeable changes are also observed in the equilibrium transmission spectra shown in Fig. 6(b). On the one hand, some transmission peaks dominated by the oxygen lone-pair states become less prominent and even disappear completely so that the number of the transmission peaks in the Au-helical-S( $\left(\mathrm{CH}_{2} \mathrm{CH}_{2} \mathrm{O}\right)_{n} \mathrm{CH}_{3}-\mathrm{Au}$ junctions with $\mathrm{n}=4$ and 5 is less than that of the oxygen atoms in the molecular backbone. On the other hand, those remarkable transmission peaks are broadened by the enhanced S-O interaction, which indirectly strengthens the coupling between the oxygen lone-pair states and the electronic states of the gold electrode. Especially, the first transmission peak below the Fermi energy decays toward $E_{\mathrm{F}}$ in a much slower manner and thus contributes to the transmission around $E_{\mathrm{F}}$. The trans-

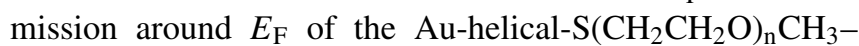
$\mathrm{Au}$ junctions is much greater than that of the $\mathrm{Au}$-trans$\mathrm{S}\left(\mathrm{CH}_{2} \mathrm{CH}_{2} \mathrm{O}\right)_{n} \mathrm{CH}_{3}-\mathrm{Au}$ junctions with $\mathrm{n}=3$, 4, and 5. When all of the non-hydrogen atoms in the molecular backbone are considered, the conductance decay constant of the Au-helical$\mathrm{S}\left(\mathrm{CH}_{2} \mathrm{CH}_{2} \mathrm{O}\right)_{n} \mathrm{CH}_{3}-\mathrm{Au}$ junctions with $\mathrm{n}=3,4$, and 5 is fitted to 
(a)

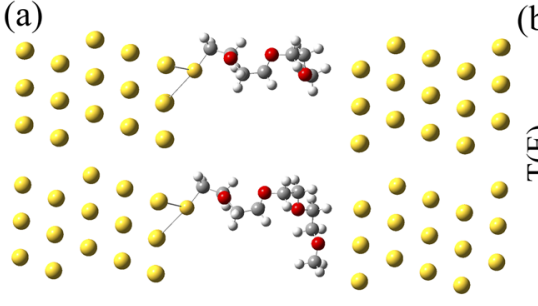

每, 0

(d)

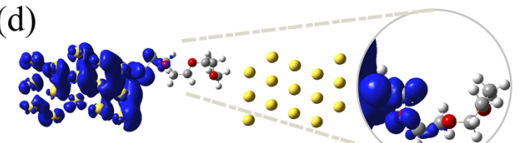

(b) 10
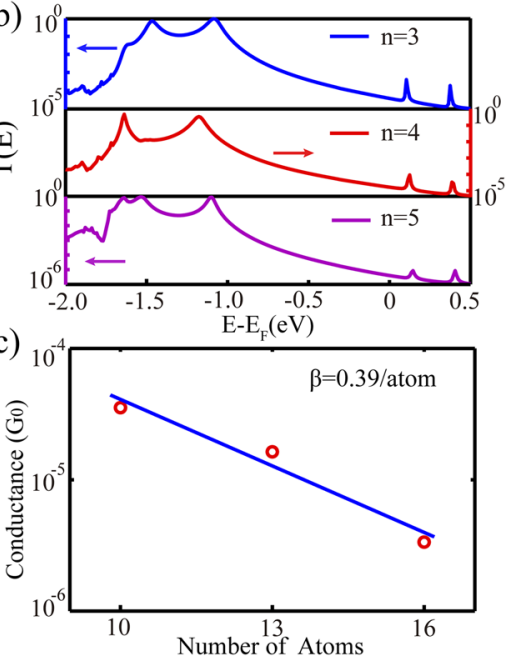

FIG. 6. The optimized atomic structure (a), the equilibrium transmission spectra (b) and the linear fit for the conductance decay constant (c) of the $\mathrm{Au}$ helical- $\mathrm{S}\left(\mathrm{CH}_{2} \mathrm{CH}_{2} \mathrm{O}\right)_{n} \mathrm{CH}_{3}-\mathrm{Au}$ junctions with $\mathrm{n}=3$, 4 , and 5, and (d) the eigenchannel of the Au-helical$\mathrm{S}\left(\mathrm{CH}_{2} \mathrm{CH}_{2} \mathrm{O}\right)_{3} \mathrm{CH}_{3}-\mathrm{Au}$ molecular junction calculated at $E_{\mathrm{F}}$ be 0.39/atom [see Fig. 6(c)], consistent with the experimental value of 0.29/atom reported by Baghbanzadeh et al. ${ }^{29}$

(a)

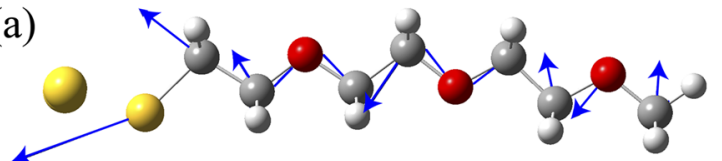

(b)

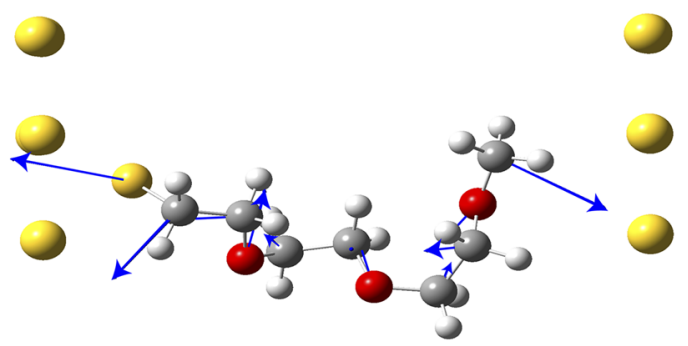

(c)

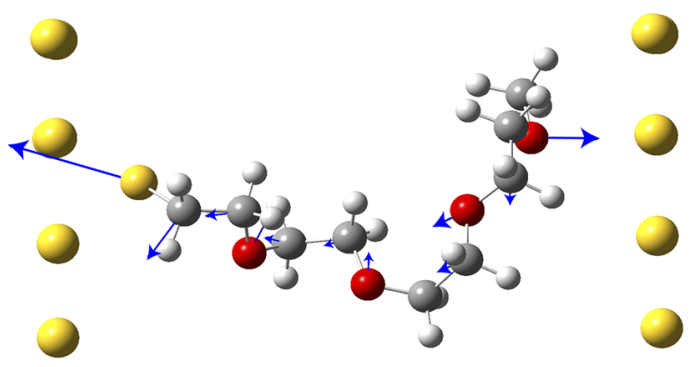

(d)

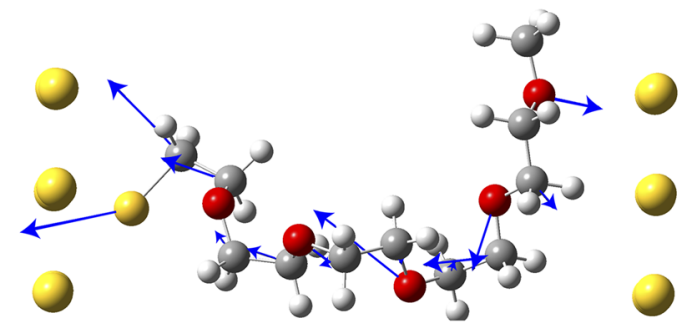

FIG. 7. Bond current profiles of the Au-trans-S( $\left.\mathrm{CH}_{2} \mathrm{CH}_{2} \mathrm{O}\right)_{3} \mathrm{CH}_{3}-\mathrm{Au}$ junction (a) and the Au-helical-S( $\left(\mathrm{CH}_{2} \mathrm{CH}_{2} \mathrm{O}\right)_{n} \mathrm{CH}_{3}-\mathrm{Au}$ junctions with $\mathrm{n}=3$ (b), 4 (c), and 5 (d). For clarity, the bond currents on $\mathrm{H}$ and $\mathrm{Au}$ atoms are not shown.
The enhanced transmission around $E_{\mathrm{F}}$ and the reduced conductance decay constant are intimately related to the

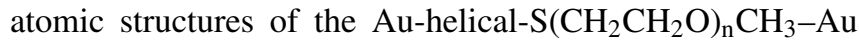
junctions. Just as shown in the eigenchannel of the $\mathrm{Au}$ helical- $\mathrm{S}_{(}\left(\mathrm{CH}_{2} \mathrm{CH}_{2} \mathrm{O}\right)_{3} \mathrm{CH}_{3}-\mathrm{Au}$ junction calculated at $E_{\mathrm{F}}$ [see Fig. 6(d)], the transmission around the Fermi energy is still dominated by the $\mathrm{Au}-\mathrm{S}$ interface hybrid state. However, when the isovalue for the eigenchannel is decreased from $2 \times 10^{-4}$ bohrs $^{-3}$ to $2 \times 10^{-5}$ bohrs $^{-3}$, we can see that some $\pi$-type orbitals of oxygen atoms participate in the transmission around $E_{\mathrm{F}}$. This is vastly different from that of the $\mathrm{Au}-$ trans- $\mathrm{S}\left(\mathrm{CH}_{2} \mathrm{CH}_{2} \mathrm{O}\right)_{n} \mathrm{CH}_{3}-\mathrm{Au}$ molecular junctions, suggesting that the twist of the oligoethers in the junctions enhances the efficiency of electron transport along the molecular backbone. Moreover, more functional groups facing the right electrode surface also improve the conductance of the Au-helical-S $\left(\mathrm{CH}_{2} \mathrm{CH}_{2} \mathrm{O}\right)_{\mathrm{n}} \mathrm{CH}_{3}-\mathrm{Au}$ junctions with $\mathrm{n}=4$ and 5 , which is evident in the bond current profile showing the pathway of electrons in the junction (see Fig. 7). ${ }^{48-50}$ Different from the $\mathrm{Au}-$ trans- $\mathrm{S}\left(\mathrm{CH}_{2} \mathrm{CH}_{2} \mathrm{O}\right)_{n} \mathrm{CH}_{3}-\mathrm{Au}$ junctions with $\mathrm{n}=3,4$, and 5 and the Au-helical- $\mathrm{S}\left(\mathrm{CH}_{2} \mathrm{CH}_{2} \mathrm{O}\right)_{3} \mathrm{CH}_{3}-$ $\mathrm{Au}$ junction where the terminal methyl group dominates the electronic coupling at the right molecule-electrode interface, the oxygen atom in the $\mathrm{CH}_{2} \mathrm{CH}_{2} \mathrm{O}$ group adjacent to the terminal methyl group not only couples more strongly with the right gold electrode but also shortens the pathway

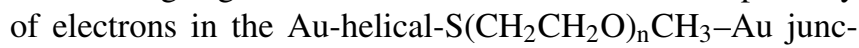
tions with $\mathrm{n}=4$ and 5 . Therefore, the conductance decay constant of 0.39/atom fitted with all of the non-hydrogen atoms in the molecular backbone is somewhat underestimated, but it only increases to $0.44 /$ atom when the terminal

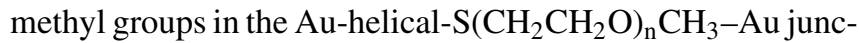
tions with $\mathrm{n}=4$ and 5 are not considered. This is still much less than that of the $\mathrm{Au}$-trans- $\mathrm{S}\left(\mathrm{CH}_{2} \mathrm{CH}_{2} \mathrm{O}\right)_{n} \mathrm{CH}_{3}-\mathrm{Au}$ junctions, demonstrating the highly efficient electron transport of the Au-helical-S( $\left.\mathrm{CH}_{2} \mathrm{CH}_{2} \mathrm{O}\right)_{\mathrm{n}} \mathrm{CH}_{3}-\mathrm{Au}$ junctions.

\section{CONCLUSIONS}

We have investigated the atomic structure and electronic transport properties of thiol-terminated oligoether molecular 
junctions employing the NEGF+DFT method. Our calculations show that the conformation of oligoethers plays a decisive role in the low-bias junction conductance. For molecular junctions with oligoethers in the trans-extended conformation, the transmission around the Fermi energy is mainly contributed by the $\mathrm{Au}-\mathrm{S}$ interface hybrid states that couple with the $\mathrm{S}-\mathrm{C}$, $\mathrm{C}-\mathrm{C}$, and $\mathrm{C}-\mathrm{O} \sigma$-bonds of the molecular backbone forming the conduction channel at $E_{\mathrm{F}}$, while the transmission peaks dominated by the $\pi$-type oxygen lone-pair states hardly affect the transmission around $E_{\mathrm{F}}$ due to their sharp peak structures and low-lying energy positions. As a result, the calculated conductance decay constant is very close to that of alkanes. By contrast, for molecular junctions with oligoethers in the helical conformation, the shortened $\mathrm{O}-\mathrm{O}$ and $\mathrm{S}-\mathrm{O}$ distances enhance their interactions and the specific spatial orientation among the oxygen and sulfur atoms results in $\sigma-\pi$ hybridization along the oligoether backbones. Therefore, some $\pi$-type oxygen orbitals contribute to the junction transmission around $E_{\mathrm{F}}$. The pathway of electrons is also shortened, and the moleculeelectrode coupling at the non-thiol side is strengthened in the Au-helical-S $\left(\mathrm{CH}_{2} \mathrm{CH}_{2} \mathrm{O}\right)_{n} \mathrm{CH}_{3}-\mathrm{Au}$ junctions with $\mathrm{n}=4$ and 5 , in which the oxygen atom of the $\mathrm{CH}_{2} \mathrm{CH}_{2} \mathrm{O}$ group neighboring the terminal methyl group interacts strongly with the right gold electrode. These two factors induced by the twist of the helical oligoethers lead to the much smaller conductance decay. Our findings provide an explanation to the discrepancy of recent experimental observations on the conductance decay constant for oligoethers and are also helpful for designing molecular wires based on heteroatom-substituted alkanethiols.

\section{ACKNOWLEDGMENTS}

This project was supported by the National Natural Science Foundation of China (Grant Nos. 61671021 and 61621061) and the National Key Research \& Development Program (Grant No. 2016YFA0201901). S.S. thanks additional funding support from the European Research Council (QUEST project) and AMBER (Science Foundation Ireland, No. 12/RC/2278).

${ }^{1}$ Z. J. Donhauser, B. A. Mantooth, K. F. Kelly, L. A. Bumm, J. D. Monnell, J. J. Stapleton, D. W. Price, Jr., A. M. Rawlett, D. L. Allara, J. M. Tour, and P. S. Weiss, Science 292, 2303 (2001).

${ }^{2}$ S. H. Choi, B. Kim, and C. D. Frisbie, Science 320, 1482 (2008).

${ }^{3}$ A. Nitzan and M. A. Ratner, Science 300, 1384 (2003).

${ }^{4}$ N. J. Tao, Nat. Nanotechnol. 1, 173 (2006).

${ }^{5}$ K. Moth-Poulsen and T. Bjørnholm, Nat. Nanotechnol. 4, 551 (2009).

${ }^{6}$ R. M. Metzger, Chem. Rev. 115, 5056 (2015).

${ }^{7}$ D. Xiang, X. Guo, and T. Lee, Chem. Rev. 116, 4318 (2016).

${ }^{8}$ T. A. Su, M. Neupane, M. L. Steigerwald, L. Venkataraman, and C. Nuckolls, Nat. Rev. Mater 1, 16002 (2016).

${ }^{9}$ L. Venkataraman, J. E. Klare, I. W. Tam, C. Nuckolls, M. S. Hybertsen, and M. L. Steigerwald, Nano Lett. 6, 458 (2006).

${ }^{10}$ F. Chen, X. Li, J. Hihath, Z. Huang, and N. Tao, J. Am. Chem. Soc. 128, 15874 (2006).

${ }^{11}$ J. M. Beebe, B. Kim, C. D. Frisbie, and J. G. Kushmerick, ACS Nano 2, 827 (2008).

${ }^{12}$ H. Song, Y. Kim, H. Jeong, M. A. Reed, and T. Lee, J. Phys. Chem. C 114, 20431 (2010).
${ }^{13}$ L. Venkataraman, J. E. Klare, C. Nuckolls, M. S. Hybertsen, and M. L. Steigerwald, Nature 442, 904 (2006).

${ }^{14}$ H. Song, Y. Kim, Y. Jang, H. Jeong, M. Reed, and T. Lee, Nature 462, 1039 (2009).

${ }^{15}$ C. R. Arroyo, S. Tarkuc, R. Frisenda, J. S. Seldenthuis, C. H. M. Woerde, R. Eelkema, F. C. Grozema, and H. S. J. van der Zant, Angew. Chem., Int. Ed. 125, 3234 (2013).

${ }^{16}$ G. Sedghi, L. J. Esdaile, H. L. Anderson, S. Martin, D. Bethell, S. J. Higgins, and R. J. Nichols, Adv. Mater. 24, 653 (2012).

${ }^{17}$ M. L. Perrin, C. J. O. Verzijl, C. A. Martin, A. J. Shaikh, R. Eelkema, J. H. van Esch, J. M. van Ruitenbeek, J. M. Thijssen, H. S. J. van der Zant, and D. Dulić, Nat. Nanotechnol. 8, 282 (2013).

${ }^{18}$ A. C. Aragonès, N. Darwish, W. J. Saletra, L. Pérez-García, F. Sanz, J. Puigmartí-Luis, D. B. Amabilino, and I. Díez-Pérez, Nano Lett. 14, 4751 (2014).

${ }^{19}$ B. Xu and N. J. Tao, Science 301, 1221 (2003).

${ }^{20}$ J. Cheng, G. Sàghi-Szabó, J. A. Tossell, and C. J. Miller, J. Am. Chem. Soc. 118, 680 (1996).

${ }^{21}$ X. Li, J. He, J. Hihath, B. Xu, S. M. Lindsay, and N. Tao, J. Am. Chem. Soc. 128, 2135 (2006)

${ }^{22}$ C. Li, I. Pobelov, T. Wandlowski, A. Bagrets, A. Arnold, and F. Evers, J. Am. Chem. Soc. 130, 318 (2008).

${ }^{23}$ G. Noy, A. Ophir, and Y. Selzer, Angew. Chem., Int. Ed. 49, 5734 (2010).

${ }^{24}$ H. Rascón-Ramos, J. M. Artés, Y. Li, and J. Hihath, Nat. Mater. 14, 517 (2015).

${ }^{25}$ A. Xiang, M. Wang, H. Wang, H. Sun, S. Hou, and J. Liao, Chem. Phys. 465-466, 40 (2016).

${ }^{26}$ L. E. Scullion, E. Leary, S. J. Higgins, and R. J. Nichols, J. Phys.: Condens. Matter 24, 164211 (2012).

${ }^{27}$ E. Wierzbinski, X. Yin, K. Werling, and D. H. Waldeck, J. Phys. Chem. B 117, 4431 (2013).

${ }^{28}$ Z. Xie, I. Bâldea, S. Oram, C. E. Smith, and C. D. Frisbie, ACS Nano 11, 569 (2017).

${ }^{29}$ M. Baghbanzadeh, C. M. Bowers, D. Rappoport, T. Żaba, L. Yuan, K. Kang, K.-C. Liao, M. Gonidec, P. Rothemund, P. Cyganik, A. Aspuru-Guzik, and G. M. Whitesides, J. Am. Chem. Soc. 139, 7624 (2017).

${ }^{30}$ F. C. Simeone, H. J. Yoon, M. M. Thuo, J. R. Barber, B. Smith, and G. M. Whitesides, J. Am. Chem. Soc. 135, 18131 (2013).

${ }^{31}$ M. Baghbanzadeh, F. C. Simeone, C. M. Bowers, K.-C. Liao, M. Thuo, M. Baghbanzadeh, M. S. Miller, T. B. Carmichael, and G. M. Whitesides, J. Am. Chem. Soc. 136, 16919 (2014).

${ }^{32}$ J. C. Cuevas and E. Scheer, Molecular Electronics: An Introduction to Theory and Experiment, 2nd ed. (World Scientific, Singapore, 2017).

${ }^{33}$ Y. Meir and N. S. Wingreen, Phys. Rev. Lett. 68, 2512 (1992).

${ }^{34}$ P. Hohenberg and W. Kohn, Phys. Rev. 136, B864 (1964).

${ }^{35}$ W. Kohn and L. J. Sham, Phys. Rev. 140, A1133 (1965).

${ }^{36}$ Y. Xue, S. Datta, and M. A. Ratner, Chem. Phys. 281, 151 (2002).

${ }^{37}$ M. brandbyge, J.-L. Mozos, P. Ordejón, J. Taylor, and K. Stokbro, Phys. Rev. B 65, 165401 (2002).

${ }^{38}$ J. Zhang, S. Hou, R. Li, Z. Qian, R. Han, Z. Shen, X. Zhao, and Z. Xue, Nanotechnology 16, 3057 (2005).

${ }^{39}$ R. Li, J. Zhang, S. Hou, Z. Qian, Z. Shen, R. Han, Z. Shen, X. Zhao, and Z. Xue, Chem. Phys. 336, 127 (2007).

${ }^{40}$ A. R. Rocha, V. M. García-Suárez, S. W. Bailey, C. J. Lambert, J. Ferrer, and S. Sanvito, Nat. Mater. 4, 335 (2005).

${ }^{41}$ A. R. Rocha, V. M. García-Suárez, S. W. Bailey, C. J. Lambert, J. Ferrer, and S. Sanvito, Phys. Rev. B 73, 085414 (2006).

${ }^{42}$ J. M. Soler, E. Artacho, J. D. Gale, A. García, J. Junquera, P. Ordejón, and D. Sánchez-Portal, J. Phys.: Condens. Matter 14, 2745 (2002).

${ }^{43}$ N. Troullier and J. L. Martins, Phys. Rev. B 43, 1993 (1991).

${ }^{44}$ J. P. Perdew, K. Burke, and M. Ernzerhof, Phys. Rev. Lett. 77, 3865 (1996).

${ }^{45}$ A. P. Sutton, Electronic Structure of Materials (Oxford University Press, New York, 1996).

${ }^{46}$ R. Li, S. Hou, J. Zhang, Z. Qian, Z. Shen, and X. Zhao, J. Chem. Phys. 125, 194113 (2006).

${ }^{47}$ M. Paulsson and M. Brandbyge, Phys. Rev. B 76, 115117 (2007).

${ }^{48}$ A. Cresti, R. Farchioni, G. Grosso, and G. P. Parravicini, Phys. Rev. B 68, 075306 (2003).

${ }^{49}$ H. Nakamura, J. Phys. Chem. C 114, 12280 (2010).

${ }^{50}$ R. Zhang, I. Rungger, S. Sanvito, and S. Hou, Phys. Rev. B 84, 085445 (2011). 